\title{
The reversible and modulation role of water- soluble gallic acid-carboxymethyl chitosan conjugates against the induced nephrotoxicity with cisplatin
}

Hani S. Hafez

Suez University

Zakaria El-Khayat

National Research Center

Ebtsam S. Kotb

Suez University

Reda F.M. Elshaarawy ( $\boldsymbol{\nabla}$ reda.elshaarawy@suezuniv.edu.eg )

Suez University

Waleed M. Serag

Suez University

Research Article

Keywords: CDDP chemotherapy, Nephrotoxicity, Gallic acid-grafted O-carboxymethyl chitosan (GA@CMLMWC), Oxidative stress

Posted Date: March 7th, 2022

DOI: https://doi.org/10.21203/rs.3.rs-1340719/v1

License: (9) (1) This work is licensed under a Creative Commons Attribution 4.0 International License. Read Full License 


\section{Abstract}

The toxicity of cisplatin (CDDP) toward the renal tubules and its severe effects on the proximal tubules limits its futher use for cancer therapy. The current study was undertaken to evaluate the protective effects of gallic acid-grafted O-carboxymethyl chitosan (GA@CMLMWC) against nephrotoxicity induced by CDDP in rats. Renal injury was assessed in the GA@CMLMWC/CDDPtreated rats using kidney injury molecule-1 (KIM-1). Moreover, the levels of reduced glutathione (GSH), malondialdehyde (MDA), and nitric oxide (NO) were measured. The comet assay was performed to measure the DNA damage. The renoprotective activity of GA@CMLMWC was supported by histo- and immuno-pathological studies of the kidney. GA@CMLMWC significantly normalized the increases in kidney homogenate of KIM-1, MDA, and NO-induced by CDDP and significantly increased GSH as compared with the CDDP group. GA@CMLMWC also significantly protects rat kidneys from CDDP-induced histo- and immuno-pathological changes. Both biochemical findings and histo- and immuno-pathological evidence showed the renoprotective potential of GA@CMLMWC against CDDP-induced oxidative stress, inflammation, and renal dysfunction in rats. In conclusion, GA@CMLMWC has been shown to mitigate the nephrotoxicity impact of CDDP in cancer therapy.

\section{Introduction}

Although the rapid progress in cancer treatment has changed the oncology landscape of treatment with decreased tumor progression and increased survival rates ${ }^{1}$, cancer patients still experience severe side effects during their chemotherapy courses, such as nephrotoxicity, neurotoxicity, ototoxicity, cardiotoxicity, hepatotoxicity, hemotoxicity, and gastrointestinal toxicity ${ }^{2}$.

Nephrotoxicity is remaining a significant complication limiting factor that lessens the efficacy of many drugs ${ }^{1}$. Nephrotoxicity produces harmful effects in the body and increases the body's ion levels, serum creatinine, serum total protein, serum urea, and blood urea nitrogen (BUN) ${ }^{3}$. According to the National Institutes of Health, there are currently over 273,000 cancer-related clinical trials underway around the world, with the majority of the tested drugs being platinumbased treatments ${ }^{4}$.

Although cisplatin (cis-diamminedichloroplatinum(II), CDDP) has been a mainstay in cancer therapy, its use is limited by two issues: the development of resistance to CDDP and the severe side effects in normal tissues caused by the high tendency to accumulate renal toxin nephrotoxins in the proximal tubules, which causes severe damage ${ }^{5}$. Notably, CDDP undergoes hydrolysis, in which one or two chloride ions are replaced by water generating positively charged electrophiles $\left[\mathrm{Pt}\left(\mathrm{NH}_{3}\right)_{2}\left(\mathrm{H}_{2} \mathrm{O}\right)_{2}\right]^{2+}$ and $\left[\mathrm{Pt}\left(\mathrm{NH}_{3}\right)_{2} \mathrm{Cl}\left(\mathrm{H}_{2} \mathrm{O}\right)\right]^{+}$that are highly reactive with nucleophilic sites

(Figure S1, ESM) ${ }^{5,6}$. These positively charged platinum complex ions are more harmful to kidney cells than CDDP because they can bind to nuclear components like DNA, RNA, and proteins to cause renal cell necrosis ${ }^{7}$. The ultimate reno-protection approaches should not only protect the kidneys but also enhance CDDP's anticancer effectiveness in chemotherapy and lessen their byproducts toxicity ${ }^{8}$. Antioxidants can preserve living tissue by either preventing the activation of oxygen for highly reactive substrates or scavenging oxidant species before they interact with 
biological targets ${ }^{9}$. Gallic acid (GA) is a natural polyphenolic antioxidant widely found in many natural products ${ }^{10,11}$. It has been found that GA and its derivatives have a wide variety of favorable benefits in the prevention and/or treatment of several illnesses. Their good safety and stability profiles also make them viable candidates for the introduction of dietary supplements ${ }^{12}$. GA's protective function is due to being a strong natural antioxidant that can inhibit reactive oxygen species (ROS), e.g., hydrogen peroxide, superoxide anions, hypochlorous acid, and hydroxyl radicals, thereby preventing oxidative stress in tissues ${ }^{13,14}$. GA has been reported to avert many disorders, including cardiovascular disease, cancer, inflammation, infection, diabetes, and neurological impairments ${ }^{15}$.

Several difficulties have been encountered in using natural antioxidants in pharmaceutical, biomedical, and food products, including volatility, instability, and oxidative conditions. Therefore, it is important to immobilize the natural antioxidants onto the surface of stable solid supports such as biopolymers and inorganic materials to overcome the aforementioned obstacles ${ }^{16}$. Chitosan (CS) as a highly used polysaccharide has received a lot of attention because of its biodegradability, biocompatibility, non-antigenicity, and non-toxicity ${ }^{17}$. Additionally, it has been proven to hold a wide range of specific properties such as antimicrobial, antitumor, antiinflammatory, antioxidant, and anti-hypercholesterolemic roles. Many disciplines, including biomedicine, pharmaceuticals, water treatment, functional membranes, and the food industry, have benefited amazing potentials for CS ${ }^{17,18}$. Noteworthy, grafting phenolic compounds has been shown to give polymers new qualities, such as improved mechanical and physiological properties and variations in solubility, antioxidant and emulsifying capabilities. In addition, the bioactivities of phenolic compounds grafted onto polymers were higher than those of the free phenolic compounds in question ${ }^{19}$. In this context, recently, more attention has been driven to biomacromolecules functionalization such as CS or any other derivative thereof with naturally occurring flavonoids such as gallic acid or caffeic acid, or catechins ${ }^{16},{ }^{20}$. Because of the distinct properties of CS and GA, as well as our ongoing pursuit of new highly effective chemotherapeutic candidates $21,22,23$. The present study aimed to investigate the biomedical and renoprotective of gallic acid-grafted $O$-carboxymethyl chitosan, additionally, to study their anti-inflammatory and antioxidant activity against the CDDP by-products in vivo.

\section{Materials And Methods}

Details for chemicals and solvents used in this work along with their suppliers were provided in the electronic supplementary material (ESM). Also, the extraction of CS from crab shells and its carboxymethylation were described in the ESM. In addition, instrumentation and different analytical techniques used for the full characterization of all synthesized materials were described in ESM.

\section{Preparation of gallic acid-grafted O-carboxymethyl chitosan (GA@CMLMWC). The}

GA@CMLMWC was prepared following the previously described protocol ${ }^{24}$ with a slight

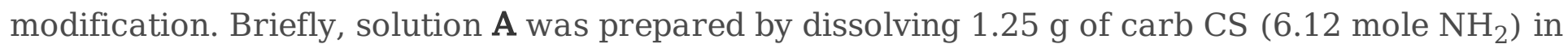
a mixed-solvents system of deionized water/methanol $(1 / 2 \mathrm{v} / \mathrm{v}, 30 \mathrm{~mL})$, then the obtained solution 
was adjusted to $\mathrm{pH} 6.8$ with trimethylamine. In solution B, Gallic acid (1.12 g; $6.58 \mathrm{mmol})$ was dissolved in $10 \mathrm{~mL}$ of methanol and mixed with a solution of dicyclohexylcarbodiimide (DCC) (0.63 g; $3.28 \mathrm{mmol})$ in methanol (10 mL) under stirring. After $1 \mathrm{~h}$, solution B was gradually added to solution $\mathbf{A}$ while stirring (150 rpm) in an ice bath for $1 \mathrm{~h}$. Thereafter, the reaction mixture was removed from the ice and stirred overnight at room temperature. Then, the content was filtered to remove dicyclohexylurea (DCU) and the filtrate was kept at $2^{\circ} \mathrm{C}$ overnight. Then, the reaction mixture was diluted with $90 \mathrm{~mL}$ of diethyl ether to precipitate the desired product. The isolated product was collected by filtration and then dissolved in deionized water (20 $\mathrm{mL}$ ) and dialyzed against deionized water using dialysis tubing of a molecular weight cut-off (12000-14000 Da) for $48 \mathrm{~h}$ with 6 times deionized water changes to ensure the complete removal of un-reacted GA. Finally, the resulting solution was lyophilized at room temperature to obtain water-soluble GA@CMLMWC.

Experimental design. Sixty-four male white albino rats (3-4 months old, 250-350 gm weight) were obtained from the Animal House of National Research Center, Dokki, Giza, Egypt. The rats were housed in groups (4/cage) until surgery, after which they were singly housed. The rats were kept with free access to standard pellet animal diet and tap water under controlled temperature (24 \pm $1^{\mathrm{o}} \mathrm{C}$ ) and 12-h light dark cycle (lights on 6:30 am), and testing was carried out during the light phase. Animals were handled according to The ARRIVE guidelines 2.0: Updated guidelines for reporting animal research ${ }^{25}$ and all procedures were approved by the National Hepatology \& Tropical Medicine Research Institute's ethics committee (NHTMRI) code: 6A-2021. All efforts were made to minimize animal pain, suffering, or discomfort as well as the number of rats used in all experiments. Animal's grouping was done as following:

- Group 1 (normal control group): without treatment.

- Group 2 (GA group): healthy rats were intraperitoneal injection (i.p.) injected with GA at a dose of $40 \mathrm{mg} / \mathrm{kg}$ body weight/day for 14 days; the dose was chosen based on the previous study according to Akomolafe et al. ${ }^{26}$.

- Group 3 (CMLMWC group): healthy rats were i.p. injected with CMLMWC (178.625 mg/kg body weight/day) for 14 days; the dose was modified from a previous study of Ibrahim, et al. 27 which stated that $\mathrm{LD}_{50}$ of carboxymethyl CS was $3.5725 \mathrm{~g} / \mathrm{kg}$ body weight in mice.

- Group 4 (GA@CMLMWC group): healthy rats were i.p. injected with GA@CMLMWC at a dose of $323.3 \mathrm{mg} / \mathrm{kg}$ body weight/day for 14 days.

- Group 5 (CDDP group): healthy rats were i.p. injected with CDDP (12 mg/kg body weight) once on the tenth day during the experimental period to induce nephrotoxicity; the dose was selected from a previous study of Dizaye ${ }^{28}$.

- Group 6 (CDDP + GA) treated group: rats have received GA in a dose of $40 \mathrm{mg} / \mathrm{kg}$ body weight/day, i.p. for 14 days with a single dose of CDDP (12 mg/kg body weight, i.p.) on the tenth day. CDDP was administered an hour after receiving the dose of GA ${ }^{29}$.

- Group 7 (CDDP + CMLMWC) treated group: the rats have received a 14-day treatment of CMLMWC in a dose of $178.625 \mathrm{mg} / \mathrm{kg}$ body weight/day, i.p. with a single dose of CDDP (12 
$\mathrm{mg} / \mathrm{kg}$ body weight, i.p.) on the tenth day. CDDP was administered an hour after receiving the dose of CMLMWC.

- Group 8 (CDDP + GA@CMLMWC) treated group: the rats were received GA@CMLMWC in a dose of $323.3 \mathrm{mg} / \mathrm{kg}$ body weight/day, i.p. for 14 days with a single dose of CDDP (12 mg/kg body weight, i.p.) on the tenth day. CDDP was administered an hour after receiving the dose of GA@CMLMWC.

All treatments were administered daily for 14 days except CDDP (a single i.p. injection on the tenth day of the treatment schedule) using a dosing schedule modified from the previous study of Kamel, et al. ${ }^{30}$. Five days post-CDDP administration ${ }^{31}$, rats were weighed and $3 \mathrm{ml}$ blood from each rat was withdrawn from the retro-orbital venous plexus of the eye using capillary tubes and collected in ethylenediaminetetraacetic acid (EDTA) tube for collecting whole blood for the comet assay. The rats were then sacrificed, and the two kidneys were quickly removed, washed with cold saline solution $(0.9 \% \mathrm{NaCl})$, and weighed. Then one kidney was homogenized as per a procedure described in the literature ${ }^{32}$ and prepared for measuring kidney injury molecule-1 (KIM-1), malondialdehyde (MDA), reduced glutathione (GSH), and nitric oxide (NO) parameters. The other kidney was fixed in a $10 \%$ neutral buffered formalin solution for histopathological examination and immunohistochemical studies of cyclooxygenase-2 (COX-2), caspase 3, and sodium-potassium adenosine triphosphatase ( $\mathrm{Na} \square / \mathrm{K} \square-A T P a s e)$ according to the manufacturer's instructions.

Body and kidneys weight changes. Changes in body and kidneys weights of rats from all groups were observed.

\section{Antioxidant assessments}

Determination of tissue lipid peroxidation. The lipid peroxides content in kidney homogenate was determined by estimation of MDA level using the method described by Ohkawa et al. ${ }^{33}$.

Determination of tissue GSH levels. Determination of GSH content was measured according to the colorimetric method ${ }^{34}$.

Assessment of nitrosative stress. The renal nitrite levels (NO) were determined as per the procedure described by Gonzalez-Barrios et al., ${ }^{35}$ with a slight modification of the Griess reagent system as stated by Sahu et al., ${ }^{36}$.

Estimation of an immunological marker for kidney damage. Quantitative determination of rat KIM-1 by enzyme-linked immunosorbent assay (ELISA) in kidney tissue homogenate was determined according to the manufacturer's instruction (SunLong Biotech Co. Ltd., China, Catalogue Number: SL0433Ra).

Assessment of DNA damage (Comet assay). The comet test was carried out following the protocol reported by Singh et al. ${ }^{37}$, which was slightly modified by Blasiak et al. ${ }^{38}$. An inverted fluorescent microscope (IX70; Olympus, Tokyo, Japan) with a $549 \mathrm{~nm}$ excitation filter and a 590 $\mathrm{nm}$ barrier filter, coupled to a video camera (Olympus), was used to analyze the slides at $40 \mathrm{X}$ 
magnification. Damaged cells took on the appearance of a comet, with a brilliantly fluorescent head and a long, brightly fluorescent tail that were separated from one another during electrophoresis.

Histopathological assessment. Retinal tissue was immediately removed and fixed in $10 \%$ neutral buffered formalin for one day before being dehydrated in increasing alcohol concentrations, purified with xylene, and then immobilized in paraffin. Afterward, paraffin blocks were cut into tissue sections $(5 \mathrm{~m})$ and stained with Hematoxylin-Eosin (H\&E) as previously described by Moradi et al. ${ }^{39}$.

Immunohistochemistry (IHC) analysis. Sections were successively treated for 30 minutes with $0.3 \% \mathrm{H}_{2} \mathrm{O}_{2}$ in PBS and $10 \%$ goat serum in PBST. After that, the sections were incubated overnight at room temperature with rabbit anti-COX2 antibodies $(1: 50 \mu \mathrm{g} / \mathrm{ml}$ Leader in Biomolecular Solutions for Life Science, Catalog No.: A1253), caspases-3 ( 1:50 $\mu \mathrm{g} / \mathrm{ml}$ Leader in Biomolecular Solutions for Life Science, Catalog No.: A11953), mouse ATPase, $(\mathrm{Na}(+) \mathrm{K}(+))$ alpha subunit (2$5 \mu \mathrm{g} / \mathrm{ml}$, Developmental Studies Hybridoma Bank (DSHB), Iowa University, USA). Finally, these sections were treated with biotinylated goat anti-mouse IgG (1:200) followed by streptavidin peroxidase complex $(1: 200)$.

Statistical analysis. Kidney sections were examined and proteins expression has been quantified using Image J software and processed in Prism 7 software (GraphPad, San Diego, CA, United States), all data have been presented as mean \pm standard errors (SE) for each protein distribution in the different areas of the kidney tissue. The IBM SPSS software package version 20.0 (Armonk, NY: IBM Corp) was used to analyze the data that was entered into the computer. Quantitative data were described using mean and SE. The significance of the obtained results was judged at the 5\% level. Statistical comparisons between experimental groups were performed using a one-way analysis of variance (ANOVA) test, followed by Tukey's multiple comparison post hoc test for pairwise comparisons. Differences with values of $p<0.05$ were considered statistically significant.

\section{Results And Discussion}

Preparation of GA@CMLMWC conjugate. Initially, CS was extracted from crab shells using our routine protocol that involved demineralization, deproteinization, and deacetylation processes ${ }^{40}$. The obtained crab CS was subjected for partial degradation mediated by hydrogen peroxide to low-molecular-weight chitosan (LMWC) which was submitted to the carboxymethylation reaction for the C6-OH group using chloroacetic acid at ambient conditions to yield $O$-carboxymethyl low molecular weight chitosan (O-CMLMWC) ${ }^{41}$ (see Scheme 1).

Eventually, the amide coupling reaction between the amino group of $O$-CMLMWC and carboxylic group of GA has been carried out to graft the surface of CMLMWC with GA, aiming fabrication of GA@CMLMWC conjugate. DCC was used as a coupling agent to activate the carboxylic group of GA and facilitate amide bond formation. Chemical modifications of CS are generally favored to modify its solubility and capacity to interact with other substances. Carboxymethyl chitosan is a water-soluble biopolymer that exhibits many superior characteristics and potentials (non-toxicity, 
biocompatibility, biodegradability, and good film-forming capability) ${ }^{42}$. Antioxidant-biopolymer conjugates could be used as new food additives, packaging materials, and biomedical materials to maximize the benefits of each constituent ${ }^{16}$.

\section{Structural characterization of GA@CMLMWC conjugate}

The synthesized materials were obtained in excellent yields and structurally characterized using the elemental and spectral analyses (FTIR, UV-Vis, NMR $\left({ }^{1} \mathrm{H},{ }^{13} \mathrm{C}\right)$ ).

Viscosity average molecular weight ( $\mathrm{M}_{\mathrm{v}}$ ), degree of deacetylation (DD), degree of polymerization (DP $\mathrm{v}$ ), carboxymethylation degree (CD), and grafting degree (GD). The $M_{\mathrm{v}}$ and $D P_{\mathrm{v}}$ of crab CS-based materials (CS, LMWC, and $O$-CMLMWC) were quantified based on the values of the intrinsic viscosities of their respective solutions in $0.10 \mathrm{M} \mathrm{NaCl}$ at $25^{\circ} \mathrm{C}$ (See $\mathbf{E S M}$ ). On the other hand, the values of DD for CS and LMWC samples were calculated from the elemental analysis and quantitative handling of the spectroscopic data (IR and ${ }^{1} \mathrm{H}-\mathrm{NMR}$ ) of them (See also ESM). The results of these experiments were collected in Table 1.

Furthermore, the values of carboxymethylation degree (CD) for the carboxymethylated LMWC ( $O$ CMLMWC) and grafting degree (GD) of GA@CMLMWC conjugate were estimated using acid-base titration and Folin-Ciocalteau method ${ }^{18}$ for CD and GD, respectively (See ESM).

Table 1

Viscometric average molecular weights $\left(M_{\mathrm{v}}\right)$, degree of polymerization $\left(D P_{\mathrm{v}}\right)$, degrees of deacetylation (DD), carboxymethylation degree (CD), and grafting degree (GD) of new materials

\begin{tabular}{|c|c|c|c|c|c|c|c|c|c|c|}
\hline \multirow[t]{2}{*}{ Sample } & \multirow{2}{*}{$\begin{array}{l}M_{V} \\
(\mathrm{kDa})\end{array}$} & \multirow[t]{2}{*}{$D P_{V}$} & \multicolumn{3}{|l|}{$\mathrm{DD} \%$} & \multicolumn{3}{|c|}{ EA Calcd (Found) (\%) } & \multirow[t]{2}{*}{$\mathrm{CD} \%$} & \multirow[t]{2}{*}{ GD\% } \\
\hline & & & $\begin{array}{l}{ }^{1} \mathrm{H}- \\
\mathrm{NMR}\end{array}$ & FTIR & EA & C & $\mathbf{H}$ & $\mathbf{N}$ & & \\
\hline CS & 463.8 & 2790 & 87.3 & 83.5 & 86.4 & 45.14 & 6.81 & 8.39 & - & - \\
\hline LMWC & 24.5 & 143 & 88.9 & 84.7 & 87.7 & (44.99) & (6.87) & (8.29) & - & - \\
\hline CMLMWC & 33.33 & 142.4 & 79.5 & 78.5 & 74.2 & 45.10 & 6.82 & 8.42 & 67.9 & - \\
\hline \multirow{5}{*}{$\begin{array}{l}\text { GA@ } \\
\text { CMLMWC }\end{array}$} & \multirow[t]{5}{*}{31.38} & \multirow[t]{5}{*}{142.2} & \multirow[t]{5}{*}{ - } & \multirow[t]{5}{*}{ - } & \multirow[t]{5}{*}{-} & $(45.03)$ & $(6.86)$ & $(8.38)$ & \multirow[t]{5}{*}{ - } & \multirow[t]{5}{*}{41.3} \\
\hline & & & & & & 42.73 & $\begin{array}{l}6.27 \\
(6.28)\end{array}$ & & & \\
\hline & & & & & & $(4$ & \multirow{3}{*}{$\begin{array}{l}(0.20) \\
(5.66)\end{array}$} & $(6.03)$ & & \\
\hline & & & & & & 44.84 & & 5.11 & & \\
\hline & & & & & & $(44.35)$ & & $(5.02)$ & & \\
\hline
\end{tabular}

Fourier-transform infrared spectroscopy (FTIR) analysis. Usually, the FTIR spectral data provide preliminary evidence for proving the success of the preparation of new materials. In this context, the FTIR spectra of GA, LMWC, O-CMLMWC, and the conjugate (GA@CMLMWC) were depicted 
in Fig. 1A. In the spectrum of GA, it can observe the characteristic bands at 3461 and $3266 \mathrm{~cm}^{-1}$ assignable for the stretching vibrations phenolic $\mathrm{O}-\mathrm{H}$ group; additional evidence for this postulate is the observation of other IR peaks at 1370 and $1272 \mathrm{~cm}^{-1}$ due to in-plane bending and vibration $\mathrm{O}-\mathrm{H}$ and Aryl-O, respectively. In addition, the stretch due to the vibration of $\mathrm{C}=\mathrm{O}$ of the carboxyl group can be observed at $1696 \mathrm{~cm}^{-1}$. Finally, the stretch observed at 3061, 1612 and $1537 \mathrm{~cm}^{-1}$ could be ascribed to stretching vibrations of $\mathrm{Ar}-\mathrm{H}$ and $\mathrm{C}=\mathrm{C}$ of a benzene ring.

The FTIR spectrum of $O$-CMLMWC displayed three characteristic bands centered at 3384, 3278, and $2904 \mathrm{~cm}^{-1}$ attributable to the stretching vibrations of alcoholic $\mathrm{O}-\mathrm{H} / 1^{\circ}$ amine $\mathrm{N}-\mathrm{H}$ and aliphatic C-H groups, respectively. The two stretches noticed at 1596 and $1417 \mathrm{~cm}^{-1}$ could be assigned to the asymmetrical and symmetrical stretching vibrations, respectively, of the $\mathrm{COO}^{-}$ group. Meanwhile, the non-sharing of the primary amine group of LMWC in carboxymethylation reaction could be confirmed from the sustaining of the emergence of the $\mathrm{N}-\mathrm{H}$ bending of a primary amine in the $O$-CMLMWC spectrum at $1596 \mathrm{~cm}^{-1}$. Moreover, the significant decrease in the intensity of the primary hydroxyl group (C6-OH) (observed at $1062 \mathrm{~cm}^{-1}$ in the LMWC spectrum) offered further evidence for $O$-carboxymethylation of CS. Notably, GA@CMLMWC conjugate exhibited a different FT-IR spectrum as compared to its native constituents. For instance, the band observed at $1596 \mathrm{~cm}^{-1}$ in the CMLMWC spectrum was significantly decreased in GA@CMLMWC spectrum as well as a new peak has emerged at $1694 \mathrm{~cm}$ - 1, which could be assigned to the stretching of the carbonyl group of amide I. The findings indicate the successful grafting of GA molecules on the surface of $O$-CMLMWC through the covalent linkage between the $\mathrm{NH}_{2}$ group of $\mathrm{O}$-CMLMWC and the $\mathrm{COOH}$ group of GA to form an amide bond. On the other hand, no vibrational band was observed in the spectral region distinctive of the absorption of the ester carbonyl group (1700-1800 $\left.\mathrm{cm}^{-1}\right)^{43}$, confirming that no ester bond was formed between the $\mathrm{OH}$ groups of the $\mathrm{CS}$ backbone and the $\mathrm{COOH}$ group of GA. Consequently, the grafting of GA on $O$ CMLMWC occurs only through the amide coupling reaction between $\mathrm{NH}_{2}$ and $\mathrm{COOH}$ of OCMLMWC and GA, respectively.

UV-Vis spectra. Figure 1B shows the UV-Vis spectra of GA, O-CMLMWC, and GA@CMLMWC conjugate. A main distinct peak can be seen in the spectrum of GA at $264.00 \mathrm{~nm}$, which could be assigned to the $\Pi \rightarrow \Pi^{*}$ transition in the benzene ring. On the other hand, the UV-Vis spectrum of LMWC displayed an intense UV peak at $225 \mathrm{~nm}$ along with a broad absorption peak at $265.50 \mathrm{~nm}$ in the UV region. Noteworthy, the chlomethylation of CMLMWC results in the almost complete disappearance of the $225 \mathrm{~nm}$ peak and the emergence of a new abroad band at $344.50 \mathrm{~nm}$ for OCMLMWC. This band could be assigned to the $n \rightarrow \Pi^{*}$ transition of the carboxyl group ${ }^{44}$. The redshift of the GA absorption peak from $264.00 \mathrm{~nm}$ to $270.50 \mathrm{~nm}$, which could be attributed to the decrease in energy required for the $\Pi \rightarrow \Pi^{*}$ and $n \rightarrow \Pi^{*}$ transitions due to the covalent linkage between O-CMLMWC and GA, is indicative of the successful grafting of GA onto O-CMLMWC. In addition, the emergence of a new UV peak at $364.00 \mathrm{~nm}$ in the spectrum of the GA@CMLMWC offers further evidence.

NMR spectroscopy. Another strong proof for the success of GA grafting onto the $O$-CMLMWC surface was provided by the ${ }^{1} \mathrm{H}$ NMR spectral technique. As shown in Figure S2 (ESM), GA 
exhibited only a sharp peak at $6.99 \mathrm{ppm}$ attributable to the resonance of phenyl protons ${ }^{45}$. On the other hand, the two peaks observed at $\delta 3.86$ and $1.83 \mathrm{ppm}$ in the NMR spectrum of LMWC

(Figure S3, ESM) could be assigned to the resonance of protons of the glucopyranose unit and the methyl group of the N-acetyl glucosamine unit, respectively, of the CS backbone ${ }^{46}$. The emergence of a new singlet peak in the spectrum of $O$-CMLMWC (Figure S4, ESM) at $64.30 \mathrm{ppm}$ equivalent for two protons of a methylene group, is indicative of the success of chloromethylation of LMWC into $O$-CMLMWC ${ }^{47}$. As for the ${ }^{1} \mathrm{H}$ NMR spectrum of GA@CMLMWC (Figure S5, ESM), the two sets of signals distinctive of GA and O-CMLMWC have been merged. For instance, in addition to the signals of the LMWC backbone, two singlet peaks at $\delta 4.29$ and $6.89 \mathrm{ppm}$ are characteristic of methylene and phenyl protons of O-CMLMWC and GA, respectively, can be seen. These findings also confirm the successful grafting of the O-CMLMWC surface by GA ${ }^{44}$.

XRD analysis. XRD analysis was used to investigate the crystallinity of GA, LMWC, O-CMLMWC, and GA@CMLMWC. GA exhibited several sharp diffraction peaks, demonstrating it was a crystalline compound. CS exhibited a characteristic peak at $2 \theta=19.70^{\circ}$, corresponding to the high crystallinity of CS. In this study, the characteristic peak of $O$-CMLMWC appears at an angle of $31.74^{\circ}$. O-CMLMWC has an amorphous structure. This difference in their structure proves that $O$-CMLMWC is more soluble than LMWC in water. The XRD pattern of GA@CMLMWC shows the major peak at $37.19^{\circ}$.

Body and kidneys weight changes results. The CDDP-treated group showed a significant decrease in the final bodyweight of the rats as compared with the control group, GA group, CMLMWC group, and GA@CMLMWC group $(\mathrm{p}<0.001)$. Also, the body weights of rats in the (CDDP + GA) group significantly decreased in comparison with the control group, CMLMWC group, and GA@CMLMWC group ( $\mathrm{p}<0.001)$, whereas in $(\mathrm{CDDP}+\mathrm{GA} @ \mathrm{CMLMWC})$ group, the treatment with GA@CMLMWC reversed body weight loss in the present study Fig. 2A. The body weight change (\%) of rats in the CDDP group significantly decreased in comparison with the control group, GA group, CMLMWC group, and GA@CMLMWC group. Also, the body weight change (\%) of rats in the (CDDP + GA) group significantly decreased in comparison with the control group, CMLMWC group, and GA@CMLMWC group, whereas in the (CDDP + GA@CMLMWC) group, the treatment with GA@CMLMWC reversed body weight loss compared with the CDDP group or (CDDP + GA) group Fig. 2B. The renal index in the CDDP group was significantly higher than that in the control group, GA group, CMLMWC group, and GA@CMLMWC group. Also, the renal index in the (CDDP + GA) group was significantly higher than that in the control group, CMLMWC group, and GA@CMLMWC group, whereas in the (CDDP + CMLMWC) group and (CDDP + GA@CMLMWC) group, the treatment with CMLMWC and GA@CMLMWC respectively decreased the index significantly compared with the CDDP group Fig. 2C.

\section{Antioxidant indices assessment}

\section{Malondialdehyde (MDA)}

Oxidative stress (OS) has been assigned as one of the primary causal elements in the toxicological consequences of the majority of chemotherapy medicines ${ }^{10}$. As a result of oxidative damage in 
nephrotoxicity, ROS causes the liberation of $\mathrm{H}$-atoms from unsaturated lipids, resulting in lipid peroxidation. This led to several alterations in the membrane structure and activities of the cell and causes DNA damage, cytotoxicity, and eventually cell death ${ }^{48}$. The current research showed that MDA levels in the kidney homogenates of rats administered intraperitoneal CDDP with 12 $\mathrm{mg} / \mathrm{kg}$ body weight have been significantly upregulated with $\mathrm{p}<0.001$ in comparison to the control group (Table 2). These data were consistent with previous work that indicated a significant increase with a P-value of $<0.001$ in MDA levels in CDDP-challenged mice ${ }^{49}$. Additionally, the highly increased MDA was due to the CDDP-induced lipid peroxidation and damage of the plasma membrane exerting oxidative stress ${ }^{48}$. Moreover, our study demonstrated that the rats treated with GA, CMLMWC, and GA@CMLMWC before and after administration of CDDP significantly mitigated the CDDP-induced nephrotoxicity and oxidative damage. Our findings were comparable to the work that was done by Nabavi et al. ${ }^{50}$ which showed that antioxidant pre-treatments might improve NaF-induced nephrotoxicity in rats by direct scavenging of free radical and/or enhancing the endogenous antioxidant systems. GA showed antioxidant effects by directly free radicals scavenging or by indirectly increasing the antioxidant enzymes activity and expression ${ }^{49,51}$. In addition, GA@CMLMWC uptake exerted excellent biological activities in comparison to those of GA or CMLMWC alone.

\subsubsection{Glutathione (GSH)}

Glutathione (GSH) is a cysteine-rich tripeptide with non-enzymatic antioxidant activity with a reactive thiol group that has reductive efficacy by interacting directly with $\mathrm{ROS}^{52}$. On one side, GSH levels in the CDDP group's kidney homogenate were significantly reduced compared to the control group $(\mathrm{P}<0.001)$ (Table 2$)$. The reduction in GSH levels caused by CDDP indicates a change in cellular redox state, implying that the cells are more susceptible to ROS ${ }^{53}$. On the other side, the treatment with GA@CMLMWC revealed the most ameliorated state for the upregulation of the GSH among CDDP administrated rats. Therefore, these results suggested that the decline in oxidative damage induced by CDDP is due to the antioxidant potential activity of GA@CMLMWC to improve the body's antioxidant defenses and better than the activity of GA or CMLMWC, indicating the antioxidant potential role was increased by the conjugation of GA on the CMLMWC backbone ${ }^{52}$. These findings were harmonized with those of a previous study that reported that grafting GA onto CS using the free radical-mediated conjugation technique increased the biological activities such as antioxidant, antimicrobial, and antibacterial activity. Biological activities of GA-grafted CS were higher than those of unmodified $\mathrm{CS}^{17}$. Our findings concurred with previous research results concerning the GA and CS grafting 26,39,48,53.

\subsubsection{Nitric Oxide (NO)}

NO plays an important role in both regulating renal hemodynamic and modulating inflammatory, proliferating responses to various stimuli ${ }^{26}$, and vasodilatation ${ }^{51}$. Inducible nitric oxide synthase (iNOS) synthesizes NO from L-arginine ${ }^{17}$, and its overproduction contributes to OS and tissue damage by interacting with superoxide to produce the deadly agent peroxynitrite ${ }^{51}$. The present research revealed that NO levels in the CDDP group were much higher than in the control group, and GA@CMLMWC treating rats ameliorated CDDP administration toxicity with highly 
significantly reduced nephrotoxicity and oxidative damage levels. Although treatment with GA or CMLMWC before and after CDDP administration lowered NO levels, the differences were not significant in comparison to the CDDP group. Whereas, normal rats given GA, CMLMWC, or GA@CMLMWC alone for 14 days did not reveal significant change for NO levels as compared to rats of the control group (Table 2). Regarding our study results, Ahn et al. ${ }^{17}$ stated that GAgrafted-CS inhibited significantly lipopolysaccharide (LPS) - stimulated NO and prostaglandin E2 (PGE2) production in macrophages by downregulation of the protein and mRNA expression of iNOS and COX-2. Thus, the restoration of NO levels after treatment of CDDP-intoxicated animals with GA@CMLMWC may be attributed to the inhibition of iNOS which produces toxic concentrations of $\mathrm{NO}^{54}$. Also, the study done by Moradi et al. ${ }^{39}$ discovered a significant decrease in nitrite levels in GA-treated rats, indicating that GA provided nephroprotection in Diclofenacexposed animals by lowering NO levels and hence nitrosative stress.

Table 2

Comparison of oxidative stress indicators and various parameters in renal tissues between different study groups

\begin{tabular}{|c|c|c|c|}
\hline & $\begin{array}{l}\text { MDA (nmole MDA/g } \\
\text { tissue) }\end{array}$ & $\begin{array}{l}\text { NO (umoles } / g \\
\text { tissue) }\end{array}$ & $\begin{array}{l}\text { GSH (pmole GSH/g } \\
\text { tissue) }\end{array}$ \\
\hline Group 1 (control) & $52.35 \pm 10.39$ & $29.97 \pm 3.03$ & $5.27 \pm 0.35$ \\
\hline Group 2 (GA) & $59.29 \pm 8.37$ & $25.03 \pm 1.74$ & $4.93 \pm 0.48$ \\
\hline Group 3 (CMLMWC) & $56.09 \pm 13.64$ & $25.45 \pm 6.17$ & $5.01 \pm 0.50$ \\
\hline Group 4 (GA@CMLMWC) & $58.61 \pm 7.33$ & $31.98 \pm 2.26$ & $5.20 \pm 0.70$ \\
\hline Group 5 (CDDP) & $165.9^{\mathrm{abcd}} \pm 21.28$ & $50.87^{\mathrm{abcd}} \pm 5.08$ & $2.43^{\mathrm{abcd}} \pm 0.38$ \\
\hline Group $6(\mathrm{CDDP}+\mathrm{GA})$ & $100.4^{\mathrm{e}} \pm 7.15$ & $37.59 \pm 4.96$ & $3.19 \pm 0.42$ \\
\hline $\begin{array}{l}\text { Group } 7 \text { (CDDP + } \\
\text { CMLMWC) }\end{array}$ & $83.33^{\mathrm{e}} \pm 8.36$ & $34.02 \pm 2.17$ & $4.12 \pm 0.13$ \\
\hline $\begin{array}{l}\text { Group } 8 \text { (CDDP + } \\
\text { GA@CMLMWC) }\end{array}$ & $69.71^{\mathrm{e}} \pm 7.51$ & $27.10^{\mathrm{e}} \pm 1.22$ & $4.42^{\mathrm{e}} \pm 0.41$ \\
\hline $\mathrm{F}$ & $10.444^{* * *}$ & $5.301^{* * *}$ & $5.014^{* * *}$ \\
\hline $\mathrm{p}$ & $<0.001^{* * *}$ & $<0.001^{* * *}$ & $<0.001^{* * *}$ \\
\hline
\end{tabular}

Data were expressed by using (Mean \pm SE.), $(n=8)$. F: F for ANOVA test, pairwise comparison between every 2 groups was done using the Post Hoc Test (Tukey). p: p-value for comparing between the different studied groups. a: Significant with Group 1 (Control group). b: Significant with Group 2 (GA group). c: Significant with Group 3 (CMLMWC group). d: Significant with Group 4 (GA@CMLMWC group). e: Significant with Group 5 (CDDP group). ***: Statistically high significant at $\mathrm{p} \leq 0.001$.

Immunological marker for kidney damage and serum markers of renal injury. Acute kidney injury (AKI) is considered one of the reported serious side-effects of platinum-based chemotherapeutics. 
However, so far there were no approved biomarkers for detecting the proximal tubular injury. In vitro and in vivo research, KIM-1 has shown to be a promising biomarker in detecting CDDPinduced renal injury ${ }^{55}$. Proximal tubular damage, renal tubular regeneration, and immunological response to nephrotoxicants have all been linked to KIM-1 expression. In the damaged kidney, KIM-1 mRNA and protein levels are extremely high. KIM-1 has been proposed as a non-invasive biomarker for proximal tubular injury in humans ${ }^{56}$. Our previous study showed that the kidney functions increased significantly $(p<0.001)$ in the CDDP group compared to the control group and GA group, as well as, the kidney functions were decreased $(p<0.001)$ in the (CDDP + GA) group compared to the CDDP group alone ${ }^{57}$. Additionally, in the current study, KIM-1 levels were considerably higher in the CDDP group and the (CDDP + GA) group in comparison to the control group. However, rats treated with GA, CMLMWC, or GA@CMLMWC before and after CDDP administration significantly decreased KIM-1 levels compared with the CDDP group. In addition, KIM-1 levels were considerably lower in the (CDDP + GA@CMLMWC) group compared with the (CDDP + GA) group as shown in Fig. 3. GA@CMLMWC, which combines the benefits of GA and CMLMWC, showed higher ability than GA and CMLMWC, respectively for minimizing CDDPinduced nephrotoxicity through enhancing oxidative status. In prospective research with 123 patients receiving platinum chemotherapeutics, Tanase et al. ${ }^{55}$ reported that urine levels of KIM1, NGAL, and cystatin C showed a statistically significant elevation on day three after treatment commencement in AKI patients.

Comet Assay analysis. DNA damage is one of the major underlying mechanisms of CDDP-induced nephrotoxicity. It limits cell division and eventually leads to apoptosis by inhibiting DNA replication. CDDP's genotoxicity has been linked to treatment termination ${ }^{58}$. The present study revealed high DNA damage in the CDDP-treated group (70 percent - 80 percent) (Fig. 4) compared to the control group ( 2 percent -5 percent), as evidenced by the increased movement of DNA from the comet's head to the tail ${ }^{59}$. The ability of CDDP to cross-link with purine bases in DNA, producing DNA damage in malignant cells, has been related to its genotoxic potential. This effect, however, is not tumor-specific and can harm normal cells ${ }^{49}$. The reported DNA damage caused by CDDP treatment in this study was in agreement with Hassan et al., ${ }^{60}$ who found a significant increase in the tail length of DNA, tail intensity (DNA percent), and tail moment in CDDP-treated rats compared to controls when using the comet assay to determine the renal genotoxic potential of CDDP. Consistently, our findings demonstrated that rats given GA, CMLMWC, or GA@CMLMWC before and after CDDP injection had less DNA damage compared to the CDDP group. In this study, the conjugation of GA onto CMLMWC improved the ability of CMLMWC to prevent DNA damage caused by CDDP.

This improvement could improve CMLMWC's ability to reduce DNA damage ${ }^{52}$. In the same concern, Boran et al. ${ }^{58}$ found that treatment with 50 M CDDP induced significantly enhanced DNA damage $(\mathrm{P}<0.05)$ on NRK-52E cells when compared to negative control. When compared to the exclusively CDDP-induced group, both $100 \mathrm{nM}$ and $200 \mathrm{nM}$ celastrol pre-treatment caused a statistically significant reduction $(\mathrm{P}<0.05)$ in DNA damage. 
Histopathological analysis. According to morphological and physiological studies, the renal tubule system is the site of the greatest CDDP damage, with the proximal tubules being the most affected. However, the administration of antioxidants has been shown to ameliorate CDDP-induced nephrotoxicity in animals ${ }^{28}$. Histopathological findings revealed structural abnormalities in the renal tissue of CDDP-treated rats, which matched the results of the biochemical examination. CDDP administration produces histological kidney abnormalities such as glomerular atrophy, tubule cell fragments, and enlarged epithelial cells in the proximal and distal convoluted tubules, according to our findings. Taking into consideration, treatment of rats with GA, CMLMWC, or GA@CMLMWC alone did not show any significant histopathological modifications in the kidney compared with the control group. In addition, treatment of the rats with GA, CMLMWC, and GA@CMLMWC before and after CDDP administration indicated significant improvement in proximal and distal convoluted tubules and glomerular atrophy, with the shape of the renal corpuscle and renal tubules appearing more or less normal (Fig. 5). This proves that the CDDP injection caused the histopathological lesions in the current investigation. The histopathological findings are also consistent with earlier research. 36,48,51,61.

\section{Immunohistochemistry analysis}

Cyclooxygenase-2 (COX-2). Immunohistochemical findings revealed structural changes in the renal tissue of CDDP-treated rats, which matched the results of the biochemical assessment and histopathological findings. We discovered that a single dose of CDDP (12 mg/kg body weight, i.p.) induces a significant increase in COX-2 immunoreactivity in the proximal tubular cells as compared to the control group (Fig. 6A-H). The protective effects of GA, CMLMWC, and GA@CMLMWC against CDDP were confirmed by immunohistochemical studies in the kidney. (Fig. 6I) showed that treating rats with GA or CMLMWC for ten days before CDDP injection and another four days after CDDP injection significantly reduced COX-2 densitometry immunohistochemistry expression when compared to the CDDP group $(\mathrm{p}<0.01)$ and that the COX-2 densitometry immunohistochemistry expression was highly significant decreased in the $(\mathrm{CDDP}+\mathrm{GA} @ \mathrm{CMLMWC})$ group when compared to the CDDP group $(\mathrm{P}<0.001)$.

The treated groups showed significant improvements in proximal and distal convoluted tubules, as well as glomerular atrophy. In comparison to the control group, we identified no significant immunohistochemistry changes in the kidneys of rats treated solely with GA, CMLMWC, or GA@CMLMWC. The number of antioxidant molecules grafted on the backbone of the polymer determines the antioxidant activity of a synthetic product ${ }^{62}$. GA@CMLMWC, which combines the benefits of both GA and CMLMWC, produced more antioxidants than either GA or CMLMWC, implying that GA@CMLMWC can reduce kidney damage and protect the kidney from nephrotoxicity.

Caspase-3. CDDP, it was discovered, stimulates both the extrinsic and intrinsic apoptotic pathways: the extrinsic pathway, which is initiated by death receptors, and the intrinsic pathway, which is concentrated on the endoplasmic reticulum and mitochondria. CDDP's apoptotic action can be mediated by a p53-dependent or p53-independent response ${ }^{63}$. The major component of apoptosis happens predominantly through a p53-dependent route that involves Bcl-2 family target 
proteins (i.e., Bax, Bcl-2) and caspase family activation ${ }^{24}$. Caspase-3, an executioner of apoptosis is considered as an index of apoptosis ${ }^{36}$. In the current investigation, the CDDP-treated rats had a significantly higher level of caspase-3 than the control rats. In comparison to the CDDP group, pre-and post-treatment with GA@CMLMWC following CDDP injection dramatically lowered caspase-3 levels in both the cortex and the medulla (Figs. 7I(A-H) \& 7 II(A-H), respectively). Treatment of rats with CMLMWC or GA@CMLMWC for ten continuous days before CDDP injection and additional 4 days after CDDP injection significantly decreased the caspase-3 densitometry immunohistochemistry protein expression as compared with the CDDP group $(\mathrm{p}<$ $0.01)$ and $(\mathrm{p}<0.001)$ respectively. The caspase-3 densitometry immunohistochemistry protein expression was highly considerably increased in the cortex as compared with medulla as seen in both the CDDP group $(p<0.001)$ and CMLMWC group $(p<0.001)$, also caspase- 3 were significantly expressed in the cortex as compared with medulla in (CDDP + GA) group $(p<0.01)$, $(\mathrm{CDDP}+\mathrm{CMLMWC})$ group $(\mathrm{p}<0.05)$, and (CDDP + GA@CMLMWC) group $(\mathrm{p}<0.05)$ (Fig. 7 III). Consistent with previous investigations Abd El-Rhman et al. ${ }^{64}$ stated that the CDDP-treated rats had a 483.7 percent rise in caspase-3 levels when compared to the control rats. In comparison to the CDDP group, pretreatment of CDDP-injected rats with Dibenzazepine dramatically lowered caspase-3 levels by $43.2 \%$.

$\mathrm{Na}^{+} / \mathrm{K}^{+}$-ATPase (Sodium potassium adenosine triphosphatase). Furthermore, CDDP therapy can change the expression of $\mathrm{Na}+/ \mathrm{K}+$-ATPase. $\mathrm{Na}+/ \mathrm{K}+$-ATPase is an enzyme found in the membranes of practically all animal cells. It is very important in cell physiology. For each ATP molecule consumed by the pump, three sodium ions are withdrawn and two potassium ions are imported, resulting in a single positive charge being exported every pump cycle. Furthermore, decreased $\mathrm{Na}+/ \mathrm{K}+$-ATPase activity may be attributed to lower amounts of its substrate (ATP), and increased membrane lipid peroxidation leads to membrane modification by ROS ${ }^{65}$. In the current study, i.p injection of CDDP causes nephrotoxicity manifested by a reduction in expression of kidney $\mathrm{Na}+/ \mathrm{K}+$-ATPase as compared to the control group. Thus, treatment with CMLMWC, or GA@CMLMWC before and after CDDP injection appeared to decrease the harmful effect of CDDP in both cortex and medulla (Figs. 8I(A-H) \& $8 \mathrm{II}(\mathbf{A}-\mathrm{H})$, respectively). Treatment of rats with CMLMWC or GA@CMLMWC for ten continuous days before CDDP injection and additional 4 days after CDDP injection significantly increased the $\mathrm{Na}+/ \mathrm{K}+$-ATPase densitometry immunohistochemistry protein expression as compared with the CDDP group $(\mathrm{p}<0.01)$ and $(\mathrm{p}<$ 0.001) respectively. Therefore, the unregulated expression levels of Na-K-ATPase not only depending upon functions as a physiological ion transporter, but it also functions as a signaling transducer leading to generation of ROS and oxidative modification of protein.

The $\mathrm{Na}^{+} / \mathrm{K}^{+}$-ATPase densitometry immunohistochemistry protein expression was highly considerably increased in the cortex as compared with medulla as seen in (CDDP + CMLMWC) group ( $\mathrm{p}<0.001$ ), also $\mathrm{Na}^{+} / \mathrm{K}^{+}$-ATPase protein was considerably expressed in the cortex as compared with medulla in the control group $(\mathrm{p}<0.01)$, GA group $(\mathrm{p}<0.01)$, CMLMWC group ( $\mathrm{p}$ $<0.05)$, GA@CMLMWC group ( $\mathrm{p}<0.05)$, and (CDDP + GA@CMLMWC) group $(\mathrm{p}<0.01)$, while there were no significant differences between cortex and medulla in both CDDP group and (CDDP + GA) group (Fig. 8 III). These results agreed with that of Alazragi 65 which revealed that rats 
were exposed to Amiodarone A when compared to the control group, supplementation produces pulmonary toxicity, as evidenced by a significant drop ( $\mathrm{p}$ 0.05) in serum value of lung $\mathrm{Na}+\mathrm{K}+-$ ATPase. Thus, using either ferulic acid or GA or a combination of the two reduced Amiodarone A harmful effects.

\section{Conclusion}

Our data showed that GA@CMLMWC has a protective effect in vivo against CDDP-induced nephrotoxicity by lowering excessive apoptosis, oxidative stress, and inflammation. COX-2 densitometry immunohistochemistry expression was dramatically reduced in rats treated with CMLMWC or GA@CMLMWC before or after CDDP administration. Caspase-3 densitometry immunohistochemistry protein expression was dramatically reduced in rats given CMLMWC or GA@CMLMWC before or after CDDP administration. The Na+/K+-ATPase densitometry immunohistochemistry protein expression was considerably elevated in rats treated with CMLMWC or GA@CMLMWC before or after CDDP injection. As a result, it will be a fascinating topic to investigate further in the future.

\section{Declarations}

\section{Acknowledgment}

We would like to thank Prof. Dr. / Abdel Razik Farrag at the National Research Centre (NRC), Giza, Dokki, Egypt, provided research facilities for this study. May Allah have mercy on him and his soul.

\section{References}

1. Małyszko, J., Kozłowska, K., Kozłowski, L. \& Małyszko, J. Nephrotoxicity of anticancer treatment. Nephrology Dialysis Transplantation. 32, 924-936 (2017).

2. Oun, R. \& Rowan, E. Cisplatin induced arrhythmia; electrolyte imbalance or disturbance of the SA node? European journal of pharmacology. 811, 125-128 (2017).

3. Xiang, X., Guo, C., Tang, C., Cai, J. \& Dong, Z. in Seminars in nephrology. 152-158 (Elsevier).

4. Duan, Z., Cai, G., Li, J. \& Chen, X. Cisplatin-induced renal toxicity in elderly people. Therapeutic advances in medical oncology. 12, 1-15, doi:https://doi.org/10.1177/1758835920923430 (2020).

5. Zhu, S., Pabla, N., Tang, C., He, L. \& Dong, Z. DNA damage response in cisplatin-induced nephrotoxicity. Archives of toxicology. 89, 2197-2205 (2015).

6. Karasawa, T. \& Steyger, P. S. An integrated view of cisplatin-induced nephrotoxicity and ototoxicity. Toxicology letters. 237, 219-227 (2015).

7. Sánchez-González, P. D., López-Hernández, F. J., López-Novoa, J. M. \& Morales, A. I. An integrative view of the pathophysiological events leading to cisplatin nephrotoxicity. Critical reviews in toxicology. 41, 803-821 (2011). 
8. Yang, Y., Liu, H., Liu, F. \& Dong, Z. Mitochondrial dysregulation and protection in cisplatin nephrotoxicity. Archives of toxicology. 88, 1249-1256 (2014).

9. Cirillo, G. et al. Polyphenol conjugates and human health: a perspective review. Critical Reviews in Food Science and Nutrition. 56, 326-337, doi:https://doi.org/10.1080/10408398.2012.752342 (2016).

10. Safaei, F. et al. Protective effects of gallic acid against methotrexate-induced toxicity in rats. Acta Chirurgica Belgica. 118, 152-160 (2018).

11. Ola-Davies, E. O., Olukole, S. G. \& Lanipekun, D. O. Gallic Acid Ameliorates Bisphenol AInduced Toxicity in Wistar Rats. Iranian Journal of Toxicology. 12, 11-18 (2018).

12. Kahkeshani, N. et al. Pharmacological effects of gallic acid in health and diseases: A mechanistic review. Iranian Journal of Basic Medical Sciences. 22, 225-237, doi:10.22038/ijbms.2019.32806.7897 (2019).

13. Kohansal, P., Rajai, N., Dehpour, A. R., Rashidian, A. \& Shafaroodi, H. The protective effect of acute pantoprazole pretreatment on renal ischemia/reperfusion injury in rats. Fundamental and clinical Pharmacology. 33, 405-411 (2019).

14. Kilic, K. et al. Protective effect of gallic acid against cisplatin-induced ototoxicity in rats. Brazilian journal of otorhinolaryngology. 85, 267-274 (2019).

15. Samad, N. \& Javed, A. Therapeutic effects of gallic acid: Current scenario. J Phytochemistry Biochem. 2, 1-6 (2018).

16. Kang, B., Vales, T. P., Cho, B.-K., Kim, J.-K. \& Kim, H.-J. Development of gallic acid-modified hydrogels using interpenetrating chitosan network and evaluation of their antioxidant activity. Molecules. 22, 1-11 (2017).

17. Ahn, C.-B. et al. Gallic acid-g-Chitosan modulates inflammatory responses in LPS-stimulated RAW264.7 cells via NF-kB, AP-1, and MAPK pathways. Inflammation. 39, 366-374, doi:10.1007/s10753-015-0258-2 (2016).

18. Liu, J., Lu, J.-f., Kan, J. \& Jin, C.-h. Synthesis of chitosan-gallic acid conjugate: Structure characterization and in vitro anti-diabetic potential. International journal of biological macromolecules. 62, 321-329 (2013).

19. Xie, M., Hu, B., Wang, Y. \& Zeng, X. Grafting of gallic acid onto chitosan enhances antioxidant activities and alters rheological properties of the copolymer. Journal of agricultural and food chemistry. 62, 9128-9136 (2014).

20. Elbehairi, S. E. I., Ismail, L. A., Alfaifi, M. Y., Elshaarawy, R. F. M. \& Hafez, H. S. Chitosan nano-vehicles as biocompatible delivering tools for a new Ag(I)curcuminoid-Gboxin analog complex in cancer and inflammation therapy. International Journal of Biological Macromolecules 165, 2750-2764, doi:https://doi.org/10.1016/j.ijbiomac.2020.10.153 (2020).

21. Serag, W. M., Zahran, F., Abdelghany, Y. M., Elshaarawy, R. F. M. \& Abdelhamid, M. S. Synthesis and molecular docking of hybrids ionic azole Schiff bases as novel CDK1 inhibitors and anti-breast cancer agents: In vitro and in vivo study. Journal of Molecular Structure 1245, 131041, doi:https://doi.org/10.1016/j.molstruc.2021.131041 (2021).

22. Elshaarawy, R. F. M., Eldeen, I. M. \& Hassan, E. M. Efficient synthesis and evaluation of bispyridinium/bis-quinolinium metallosalophens as antibiotic and antitumor candidates. Journal 
of Molecular Structure 1128, 162-173, doi:https://doi.org/10.1016/j.molstruc.2016.08.059 (2017).

23. Elshaarawy, R. F. M. \& Janiak, C. Antibacterial susceptibility of new copper(II) N-pyruvoyl anthranilate complexes against marine bacterial strains - In search of new antibiofouling candidate. Arabian Journal of Chemistry 9, 825-834, doi:https://doi.org/10.1016/j.arabjc.2015.04.010 (2016).

24. Ryu, B. et al. Characterization of the in vitro effects of gallic acid-graftedchitooligosaccharides in the suppression of AGS human gastric cancer cell proliferation. RSC advances. 7, 24561-24568 (2017).

25. Percie du Sert, N. et al. The ARRIVE guidelines 2.0: Updated guidelines for reporting animal research. Journal of Cerebral Blood Flow \& Metabolism 40, 1769-1777 (2020).

26. Akomolafe, S. F., Akinyemi, A. J. \& Anadozie, S. O. Phenolic acids (gallic and tannic acids) modulate antioxidant status and cisplatin induced nephrotoxicity in rats. International scholarly research notices. 2014, 1-8, doi:http://dx.doi.org/10.1155/2014/984709 (2014).

27. Ibrahim, H., El-Zairy, E. \& Mosaad, R. Preparation, characterization and median lethal dose (LD50) of carboxymethyl chitosan as target Drug Delivery. International Journal. 3, 865-873 (2015).

28. Dizaye, K. Alleviation of cisplatin-induced nephrotoxicity in rats by aqueous extract of Salvia officinalis leaves. Iraqi Journal of Pharmacy. 12, 48-55, doi:10.33899/iphr.2012.62343 (2012).

29. Ma, Z.-N. et al. Nephroprotective effects of saponins from leaves of Panax quinquefolius against cisplatin-induced acute kidney injury. International Journal of Molecular Sciences. 18, 1-18 (2017).

30. Kamel, K. M. et al. Protective effects of onion oil and selenium against cisplatin-induced nephrotoxicity and oxidative stress in rats. The Egyptian Journal of Hospital Medicine. 58, 1825 (2015).

31. Mostafa, R. E., Saleh, D. O. \& Mansour, D. F. Cisplatin-induced nephrotoxicity in rats: modulatory role of simvastatin and rosuvastatin against apoptosis and inflammation. JAPS. 8, 43-50 (2018).

32. Hussein, J., El-matty, D. A., El-Khayat, Z. \& Abdel-Latif, Y. Therapeutic Role of Coenzyme Q10 in Brain Injury during Experimental Diabetes. Journal of Applied Pharmaceutical Science. 3, 213-217, doi:10.7324/JAPS.2013.3636 (2013).

33. Ohkawa, H., Ohishi, N. \& Yagi, K. Assay for lipid peroxides in animal tissues by thiobarbituric acid reaction. Analytical biochemistry. 95, 351-358 (1979).

34. Ellman, G. L. Tissue sulfhydryl groups. Archives of biochemistry and biophysics. 82, 70-77 (1959).

35. Gonzalez-Barrios, J. A., Escalante, B., Valdes, J., Leon-Chavez, B. A. \& Martinez-Fong, D. Nitric oxide and nitric oxide synthases in the fetal cerebral cortex of rats following transient uteroplacental ischemia. Brain research. 945, 114-122, doi:https://doi.org/10.1016/S00068993(02) 02746-4 (2002).

36. Sahu, B. D. et al. Carnosic acid attenuates renal injury in an experimental model of rat cisplatin-induced nephrotoxicity. Food and Chemical Toxicology. 49, 3090-3097 (2011). 
37. Singh, N. P., McCoy, M. T., Tice, R. R. \& Schneider, E. L. A simple technique for quantitation of low levels of DNA damage in individual cells. Experimental cell research. 175, 184-191 (1988).

38. Blasiak, J. et al. Free radical scavengers can differentially modulate the genotoxicity of amsacrine in normal and cancer cells. Mutation Research/Genetic Toxicology Environmental Mutagenesis. 535, 25-34, doi:https://doi.org/10.1016/S1383-5718(02)00289-9 (2003).

39. Moradi, A. et al. Gallic Acid Exerts Nephroprotective, Anti-Oxidative Stress, and AntiInflammatory Effects Against Diclofenac-Induced Renal Injury in Malerats. Archives of medical research. 52, 380-388 (2021).

40. Elshaarawy, R. F., Abd El-Azim, H., Hegazy, W. H., Mustafa, F. H. \& Talkhan, T. A. Poly (ammonium/pyridinium)-chitosan Schiff base as a smart biosorbent for scavenging of $\mathrm{Cu}^{2+}$ ions from aqueous effluents. Polymer Testing. 83, 1-27, doi:https://doi.org/10.1016/j.polymertesting.2019.106244 (2020).

41. Sofy, A. R. et al. Mitigating effects of Bean yellow mosaic virus infection in faba bean using new carboxymethyl chitosan-titania nanobiocomposites. International Journal of Biological Macromolecules. 163, 1261-1275 (2020).

42. Shariatinia, Z. Carboxymethyl chitosan: Properties and biomedical applications. International journal of biological macromolecules. 120, 1406-1419 (2018).

43. George, W., Houston, T. \& Harris, W. Vibrational spectra and structure of esters-I. Infrared and Raman spectra of CH3COOCH3, CH3COOCD3, CD3COOCH3 and CD3COOCD3. Spectrochimica Acta Part A: Molecular Spectroscopy 30, 1035-1057 (1974).

44. Bai, R., Yong, H., Zhang, X., Liu, J. \& Liu, J. Structural characterization and protective effect of gallic acid grafted O-carboxymethyl chitosan against hydrogen peroxide-induced oxidative damage. International journal of biological macromolecules 143, 49-59 (2020).

45. Chauhdary, R. \& Singh, S. Studies in Oxidation of Gallic Acid. Asian Journal of Chemistry 7, 99 (1995).

46. Elbehairi, S. E. I., Ismail, L. A., Alfaifi, M. Y., Elshaarawy, R. F. \& Hafez, H. S. Chitosan nanovehicles as biocompatible delivering tools for a new Ag (I) curcuminoid-Gboxin analog complex in cancer and inflammation therapy. International Journal of Biological Macromolecules 165, 2750-2764 (2020).

47. Sofy, A. R. et al. Mitigating effects of Bean yellow mosaic virus infection in faba bean using new carboxymethyl chitosan-titania nanobiocomposites. International Journal of Biological Macromolecules 163, 1261-1275, doi:https://doi.org/10.1016/j.ijbiomac.2020.07.066 (2020).

48. Un, H. et al. Phloretin and phloridzin guard against cisplatin-induced nephrotoxicity in mice through inhibiting oxidative stress and inflammation. Life Sciences. 266, 1-9 (2021).

49. Lahmar, A., Dhaouefi, Z., Khlifi, R., Sioud, F. \& Ghedira, L. C. Pituranthos chloranthus oil as an antioxidant-based adjuvant therapy against cisplatin-induced nephrotoxicity. Journal of Toxicology. 2020, 1-8 (2020).

50. Nabavi, S. M. et al. Protective effect of gallic acid isolated from Peltiphyllum peltatum against sodium fluoride-induced oxidative stress in rat's kidney. Molecular and cellular biochemistry. 372, 233-239 (2013). 
51. Ghaznavi, H. et al. Ameliorative effects of gallic acid on gentamicin-induced nephrotoxicity in rats. Journal of Asian natural products research. 20, 1182-1193, doi:https://doi.org/10.1080/10286020.2017.1384819 (2018).

52. Lee, D.-S. et al. Antibacterial and synergic effects of gallic acid-grafted-chitosan with $\beta$ lactams against methicillin-resistant Staphylococcus aureus (MRSA). Canadian journal of microbiology. 60, 629-638 (2014).

53. Koyuncu, I., Kocyigit, A., Gonel, A., Arslan, E. \& Durgun, M. The protective effect of naringenin-oxime on cisplatin-induced toxicity in rats. Biochemistry research international. 2017, 1-9 (2017).

54. Khadrawy, Y. A., Hosny, E. N., El-Gizawy, M. M., Sawie, H. G. \& Ezz, H. S. A. The Effect of Curcumin Nanoparticles on Cisplatin-Induced Cardiotoxicity in Male Wistar Albino Rats. Cardiovascular Toxicology. 21, 433-443 (2021).

55. Tanase, D. M. et al. The predictive role of the biomarker kidney molecule-1 (KIM-1) in acute kidney injury (AKI) cisplatin-induced nephrotoxicity. International journal of molecular sciences. 20, 1-20 (2019).

56. Kim, S. Y. \& Moon, A. Drug-induced nephrotoxicity and its biomarkers. Biomolecules and therapeutics. 20, 268-272 (2012).

57. Kotb, E. S., Serag, W. M., Elshaarawy, R. F., Hafez, H. S. \& Elkhayat, Z. The protective role of Gallic acid in Cisplatin nephrotoxicity. Frontiers in Scientific Research and Technology. 2, 4852, doi:10.21608/fsrt.2021.77860.1040 (2021).

58. Boran, T., Gunaydin, A., Jannuzzi, A. T., Ozcagli, E. \& Alpertunga, B. Celastrol pretreatment as a therapeutic option against cisplatin-induced nephrotoxicity. Toxicology research. 8, 723730, doi:https://doi.org/10.1039/c9tx00141g (2019).

59. Shruthi, S. \& Shenoy, K. B. Gallic acid: A promising genoprotective and hepatoprotective bioactive compound against cyclophosphamide induced toxicity in mice. Environmental Toxicology. 36, 123-131 (2021).

60. Hassan, H. A., Edrees, G. M., El-Gamel, E. M. \& El-Sayed, E. A. Amelioration of cisplatininduced nephrotoxicity by grape seed extract and fish oil is mediated by lowering oxidative stress and DNA damage. Cytotechnology. 66, 419-429, doi:10.1007/s10616-013-9589-8 (2014).

61. Bazmandegan, G. et al. Calcium dobesilate prevents cisplatin-induced nephrotoxicity by modulating oxidative and histopathological changes in mice. Naunyn-Schmiedeberg's Archives of Pharmacology. 394, 515-521 (2021).

62. Park, H.-H. et al. Characterization and biological activity of PVA hydrogel containing chitooligosaccharides conjugated with gallic acid. Carbohydrate polymers. 198, 197-205 (2018).

63. Hussein, R. M. \& Al-Dalain, S. M. Betaine downregulates microRNA 34a expression via a p53dependent manner in cisplatin-induced nephrotoxicity in rats. Journal of Biochemical and Molecular Toxicology. 35, 1-9, doi:https://doi.org/10.1002/jbt.22856 (2021).

64. Abd El-Rhman, R. H., El-Naga, R. N., Gad, A. M., Tadros, M. G. \& Hassaneen, S. K. Dibenzazepine attenuates against cisplatin-induced nephrotoxicity in rats: involvement of 
NOTCH pathway. Frontiers in Pharmacology. 11, 1-11, doi:10.3389/fphar.2020.567852 (2020).

65. Alazragi, R. Protective Role of Ferulic acid and/or Gallic acid Against Pulmonary Toxicity Induced by Amiodarone in Rats. Archives of Pharmacy Practice. 11, 83-90 (2020).

\section{Scheme}

Scheme 1 is available in supplementary section.

Figures
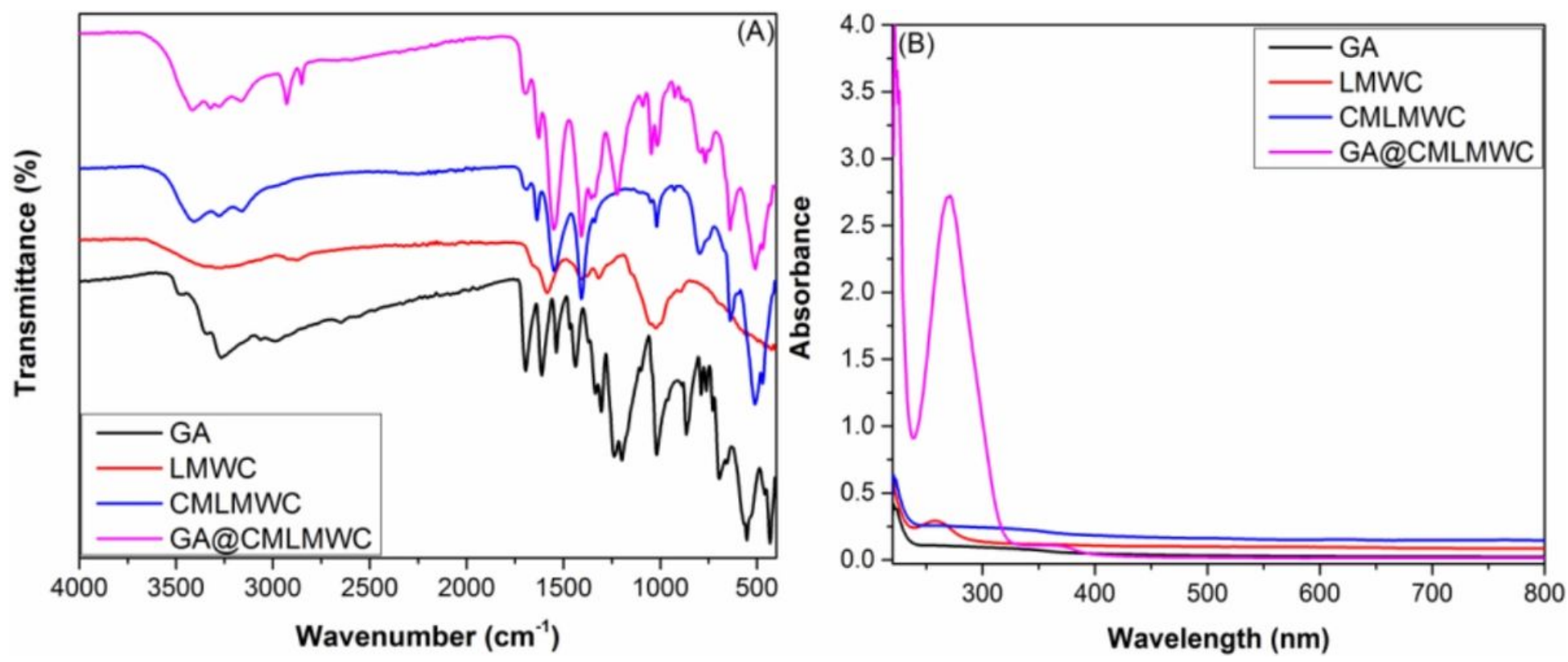

Figure 1

(A) Collective FTIR spectra of GA, LMWC, O-CMLMWC, and the conjugate (GA@CMLMWC) for comparison of their respective characteristic vibration bands. (B) UV-Vis spectra of the GA, LMWC, $O$-CMLMWC, and GA@CMLMWC in aqueous acetic acid $(0.5 \%, \mathrm{v} / \mathrm{v})(0.5 \mathrm{mg} / \mathrm{mL})$. 

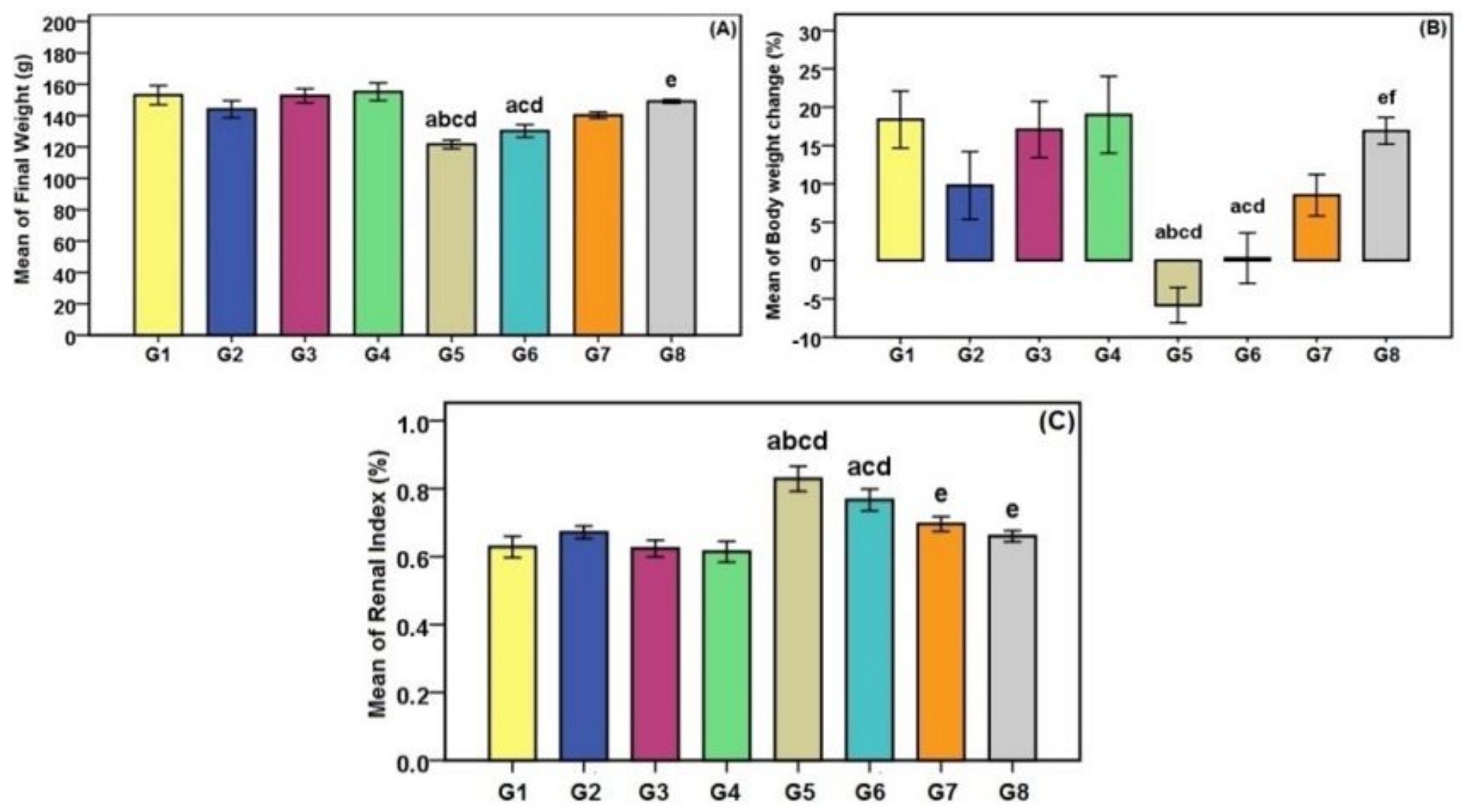

Figure 2

The effect of different treatments in CDDP-induced nephrotoxicity on (A) kidney weight of rats (B) body weight change (\%) of rats, and (C) renal index of rats in experimental groups. G1, Control; G2, GA; G3, CMLMWC; G4, GA@CMLMWC; G5, CDDP; G6, CDDP+GA; G7, CDDP+CMLMWC; G8, CDDP+GA@CMLMWC. Values are means \pm SE $(n=8)$. Data were analyzed by a one-way ANOVA test followed by Tukey's post hoc test for multiple comparisons. ${ }^{a} \mathrm{p}<0.001$ versus control group (G1), ${ }^{b} p<0.001$ vs. GA (G2), ${ }^{c} p<0.001$ vs. CMLMWC group (G3), ${ }^{d} p<0.001$ vs. GA@CMLMWC group (G4). ${ }^{\mathrm{e}} \mathrm{p}<0.001$ versus the CDDP group (G5), ${ }^{\mathrm{f}} \mathrm{p}<0.001$ versus the $(\mathrm{CDDP}+\mathrm{GA})$ group (G6). 


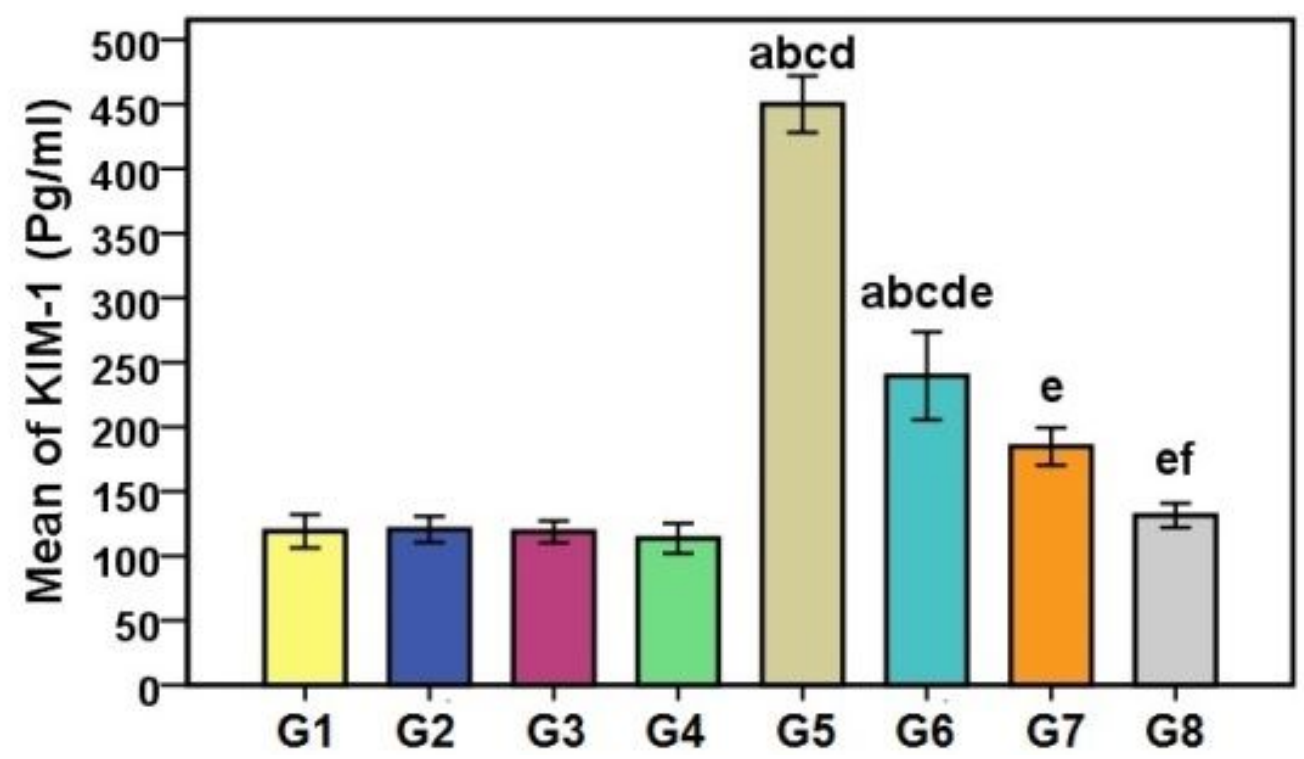

Figure 3

The effect of different treatments on KIM-1 levels in CDDP-induced nephrotoxicity in all the studied groups.Notes: Values are means \pm SE $(n=8)$. Data were analyzed by a one-way ANOVA test followed by Tukey's post hoc test for multiple comparisons. ${ }^{\mathrm{a}} \mathrm{p}<0.001$ versus control group (G1), ${ }^{b} p<0.001$ vs. GA (G2), ${ }^{c} p<0.001$ vs. CMLMWC group (G3), ${ }^{d} p<0.001$ vs. GA@CMLMWC group (G4). ${ }^{e} \mathrm{p}<0.001$ versus the CDDP group (G5), ${ }^{\text {f }}<<0.001$ versus the (CDDP+GA) group (G6). 


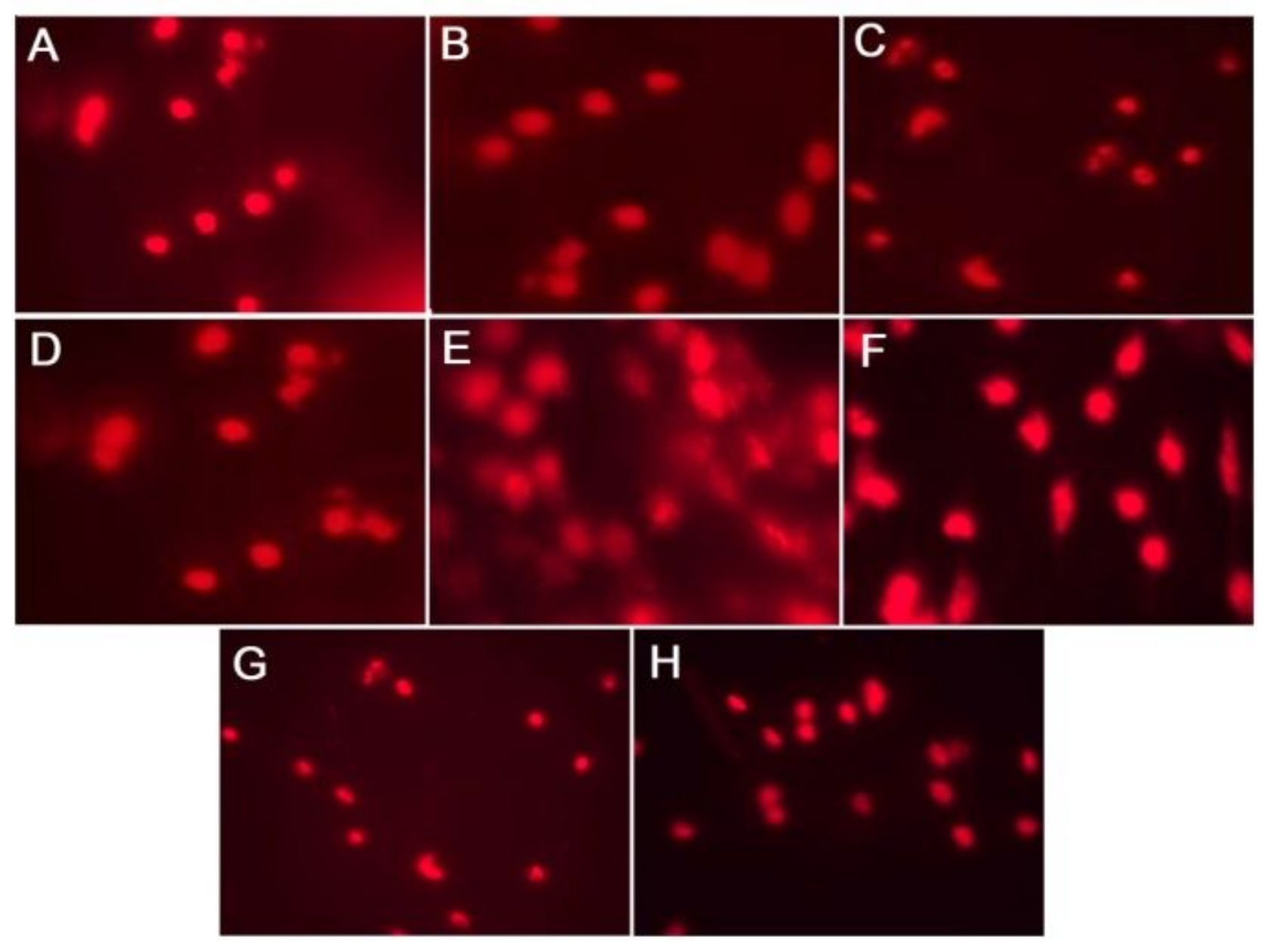

Figure 4

Comet assay showing the percentage of DNA damage in rats subjected to different treatments in CDDP-induced nephrotoxicity..(A) the control group showed a small percentage of DNA damage (2\% - 5\%). (B) the GA group showed (6\%- 15\%) DNA damage. (C) the CMLMWC group showed (7\%-13\%) DNA damage. (D) the GA@CMLMWC group showed (5\%-11\%) DNA damage. (E) high DNA damage (70\%-80\%) was observed in the CDDP group compared with the control group. (F) the (CDDP+GA) group revealed reduced DNA damage compared with the CDDP group (30\%-40\%). (G) the (CDDP+CMLMWC) group revealed reduced DNA damage compared with the CDDP group (20\%-30\%). (H) the (CDDP+GA@CMLMWC) group revealed reduced DNA damage compared with the CDDP group (27\%-30\%). 

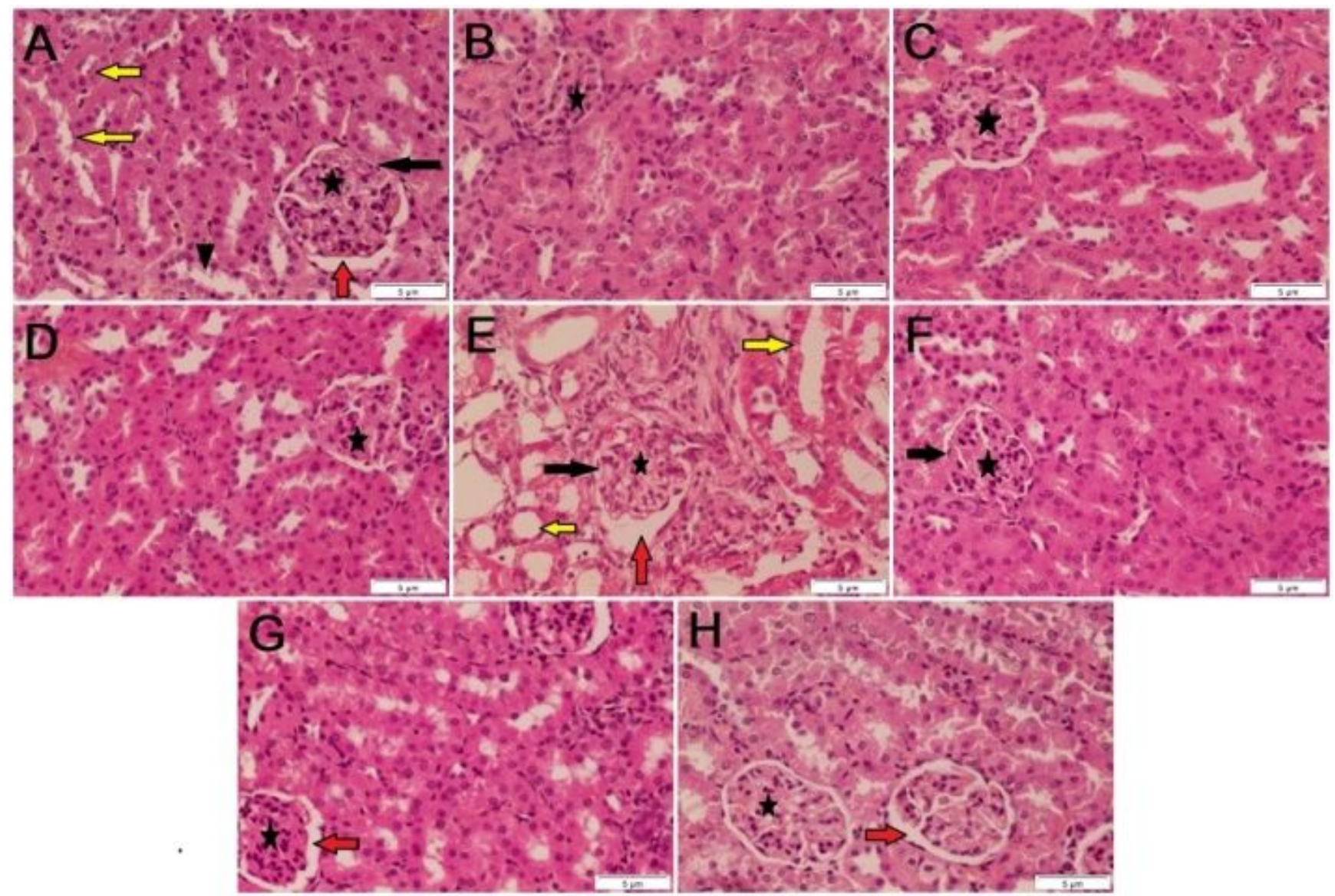

Figure 5

Histological evaluations of kidney sections in all studied groups. The control group (A) showed a normal structure of the renal corpuscle (asterisk) and renal tubules, proximal convoluted tubules (yellow arrow), and distal convoluted tubules (arrowhead). Notice the glomerulus (asterisk), urinary space (red arrow), and Bowman's capsule (black arrow). The GA group (B), the CMLMWC group (C), and the GA@CMLMWC group (D) all show a normal structure of the renal corpuscle (asterisk) and renal tubules. The CDDP group (E) showed the cortical tubules have become shrunken and atrophic and have relatively expanded interstitial spaces. Fibrosis and atrophy of the glomerulus are seen. Notice the glomerular basement membrane is diffusely and fairly uniformly thickened. The (CDDP+GA) group (F), the (CDDP+CMLMWC) group (G), and the (CDDP+GA@CMLMWC) group (H) show the structure of the renal corpuscle (asterisk) and renal tubules appearing more or less like normal. (H \& E stain, scale bar: $5 \mu \mathrm{m}$ ). 


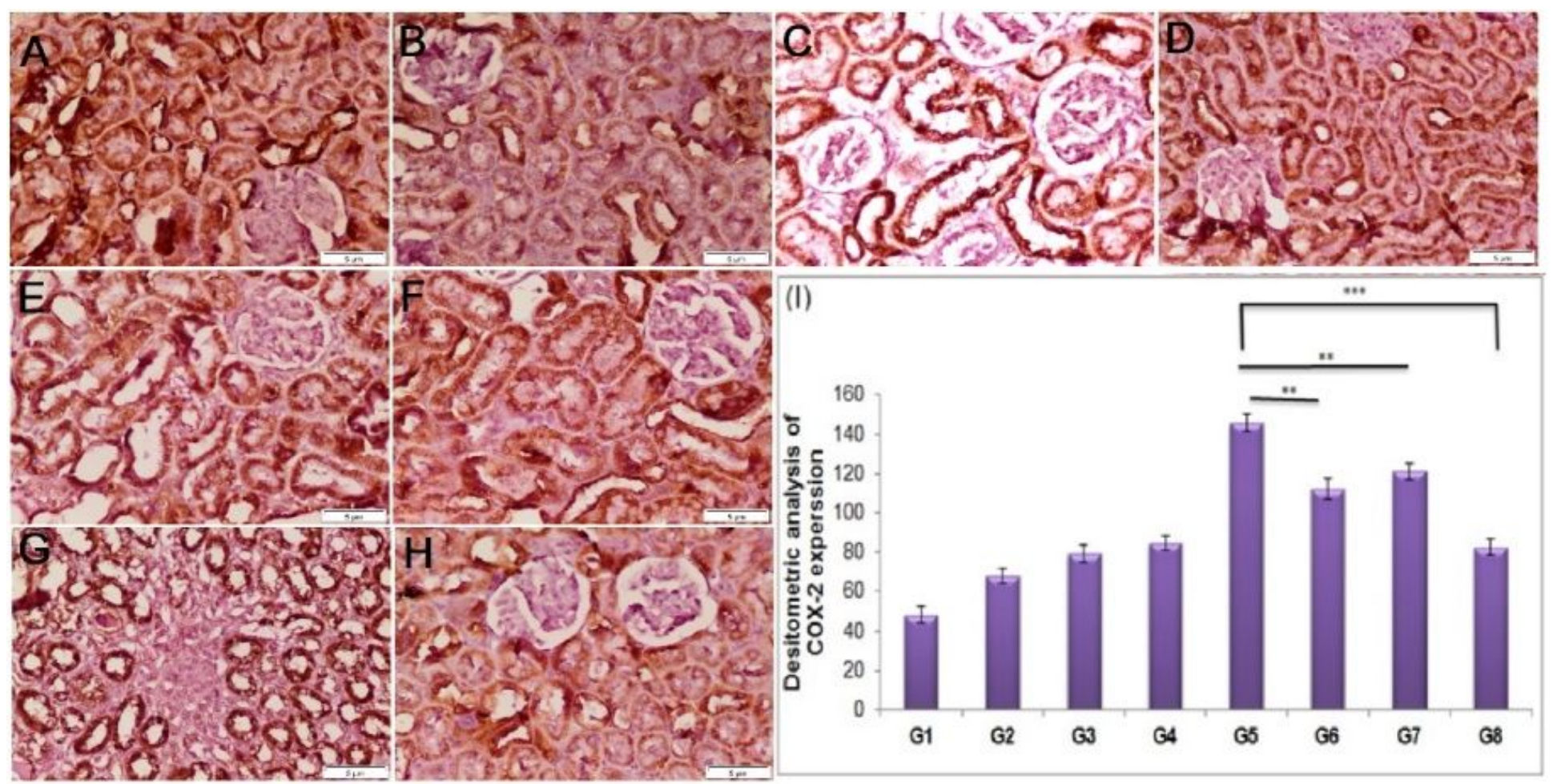

Figure 6

The effect of different treatments on COX-2 immunoreactivity and densitometry in CDDP-induced nephrotoxicity in all the studied groups. (A - H) COX-2 immunoreactivity evaluations in the kidney of rats. (I) COX-2 densitometry immunohistochemistry expression analysis in the medulla in all studied groups. The control group (A), the GA group (B), the CMLMWC group (C), and the GA@CMLMWC group (D) showed a pale stain in the proximal tubular cells and pale stain in the distal convoluted tubules and glomeruli. The CDDP group (E) showed intense COX-2 immunoreactivity in the proximal tubular cells as compared with the control. The (CDDP+GA) group (F), and the (CDDP+CMLMWC) group (G) showed moderately decreased in the COX-2 immunoreactivity in the proximal convoluted tubules as compared with the CDDP group. The (CDDP+GA@CMLMWC) group $(\mathrm{H})$ showed a pale stain in the proximal convoluted tubules as compared with the CDDP group. (COX-2 expression, scale bar: $5 \mu \mathrm{m}$ ). All data have been presented as mean $\pm \mathrm{SE} .{ }^{* *} \mathrm{p}<0.01$, and ${ }^{* * *} \mathrm{p}<0.001$. 
(I)

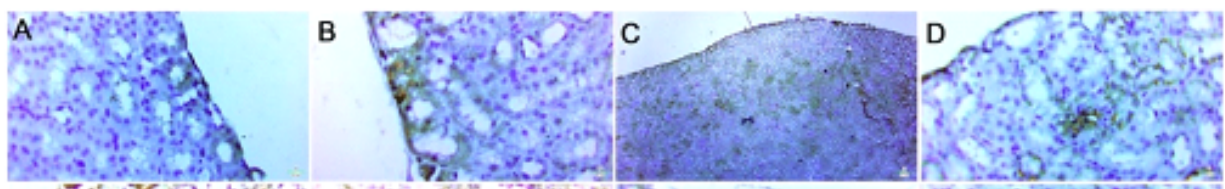

(II)

(III)
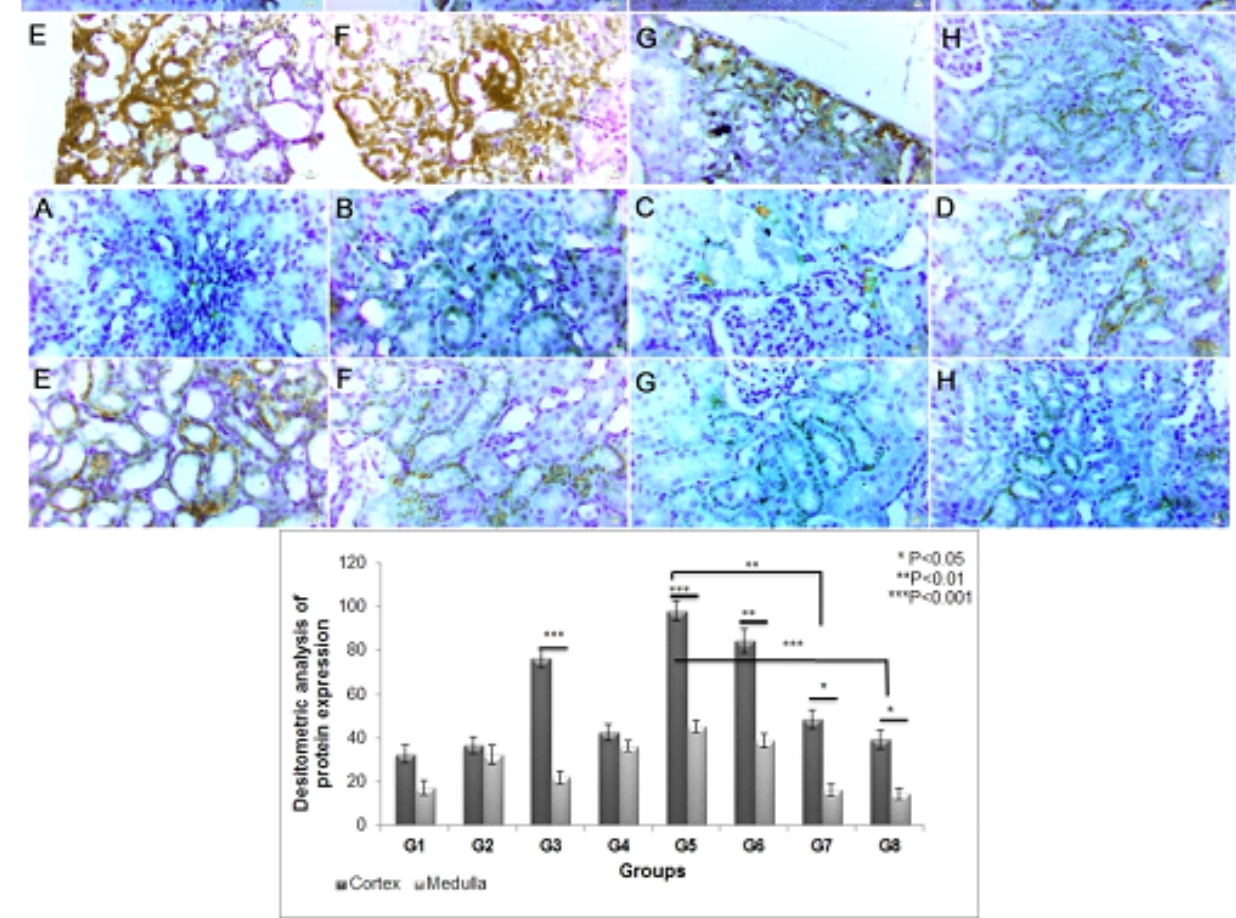

Figure 7

The effect of different treatments on caspase-3 immunoreactivity and densitometry in CDDPinduced nephrotoxicity in all the studied groups. Caspase-3 immunoreactivity evaluations in the cortex (I) and the medulla (II) of the kidney of rats. (III) caspase-3 densitometry immunohistochemistry expression analysis in both cortex and medulla in all studied groups. Caspase-3 expression using immunohistochemical staining in kidney sections: (A) the control group, (B) the GA group, (C) the CMLMWC group, (D) the GA@CMLMWC group, (E) the CDDP group, $(\mathrm{F})$ the (CDDP+GA) group, $(\mathrm{G})$ the (CDDP+CMLMWC) group, and $(\mathrm{H})$ the (CDDP+GA@CMLMWC) group. (Caspase-3 expression, scale bar: 50 um). All data have been presented as mean \pm SE. ${ }^{*} p<0.05,{ }^{* *} p<0.01$, and ${ }^{* * *} p<0.001$. 
(I)

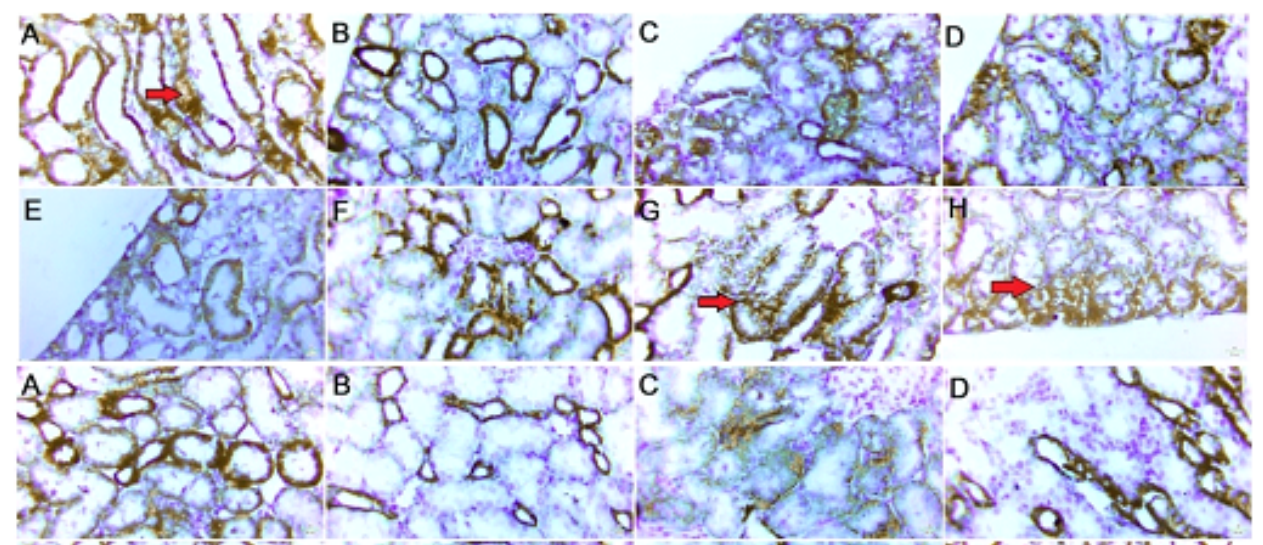

(II)

(III)
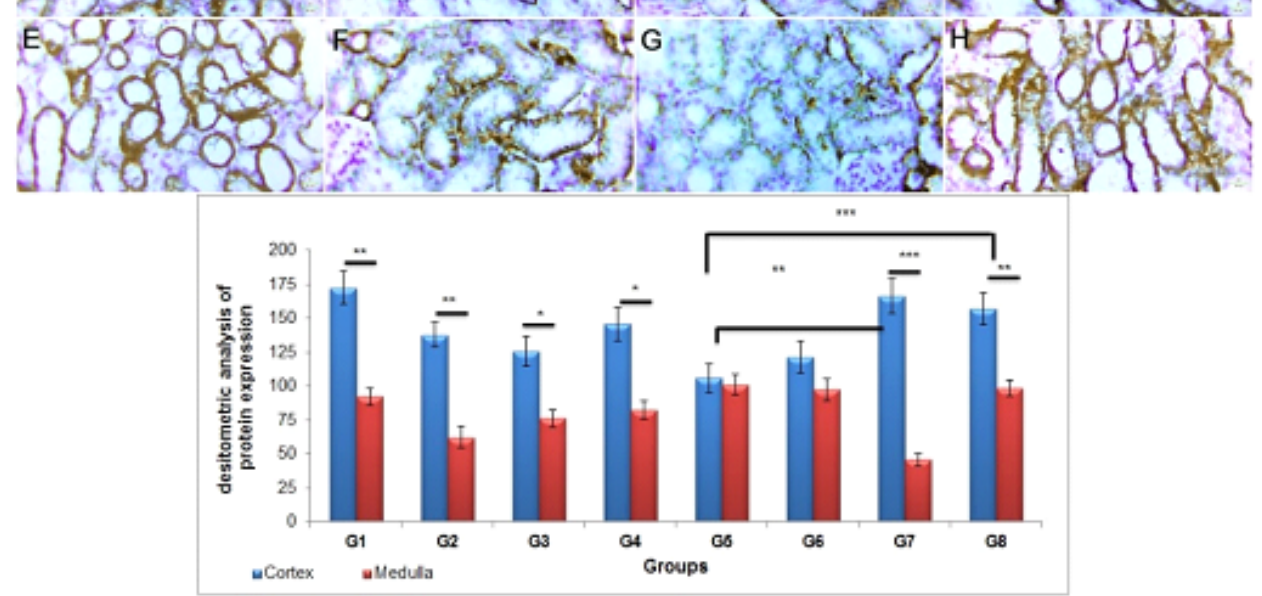

Figure 8

The effect of different treatments on $\mathrm{Na}^{+} / \mathrm{K}^{+}$-ATPase immunoreactivity and densitometry in CDDPinduced nephrotoxicity in all the studied groups. (A) a micrograph of a section of the kidney showing $\mathrm{Na}^{+} / \mathrm{K}^{+}$-ATPase immunoreactivity in the cortex. (B) a micrograph of a section of the kidney showing $\mathrm{Na}^{+} / \mathrm{K}^{+}$-ATPase immunoreactivity in the medulla. (C) $\mathrm{Na}^{+} / \mathrm{K}^{+}$-ATPase densitometry immunohistochemistry protein expression analysis in both cortex and medulla. Micrograph of the kidney section of (A) the control group, (B) the GA group, (C) the CMLMWC group, (D) the GA@CMLMWC group, (E) the CDDP group, (F) the (CDDP+GA) group, (G) the $(\mathrm{CDDP}+\mathrm{CMLMWC})$ group, and $(\mathrm{H})$ the (CDDP+GA@CMLMWC) group. $\left(\mathrm{Na}^{+} / \mathrm{K}^{+}\right.$-ATPase expression, scale bar: $50 \mathrm{um}$ ). All data have been presented as mean $\pm \mathrm{SE} .{ }^{*} \mathrm{p}<0.05,{ }^{* *} \mathrm{p}<0.01$, and ${ }^{* * *} \mathrm{p}<0.001$.

\section{Supplementary Files}

This is a list of supplementary files associated with this preprint. Click to download.

- GACMCESMSR2022F.pdf

- floatimage1.jpeg 